\title{
Hypertension and Cardiovascular Morbidity Following Surgery for Kidney Cancer
}

\author{
Umberto Capitanio $^{a, *}$, Alessandro Larcher ${ }^{a, b, c}$, Francesco Cianflone $^{a}$, Francesco Trevisani $^{a}$, \\ Alessandro Nini ${ }^{a}$, Alexandre Mottrie ${ }^{b, c}$, Andrea Mari $^{d}$, Riccardo Campi $^{d}$, Riccardo Tellini ${ }^{e}$, \\ Alberto Briganti ${ }^{a}$, Alessandro Veccia ${ }^{e}$, Hendrik Van Poppel $^{f}$, Marco Carini $^{d}$, Claudio Simeone $^{e}$, \\ Andrea Salonia ${ }^{a}$, Andrea Minervini $^{d}$, Alessandro Antonelli $^{e}$, Francesco Montorsi $^{a}$, \\ Roberto Bertini ${ }^{a}$
}

${ }^{a}$ Unit of Urology, Division of Experimental Oncology, Urological Research Institute (URI), IRCCS Ospedale San Raffaele, Milan, Italy; ${ }^{\mathrm{b}}$ ORSI Academy, Melle, Belgium; ${ }^{\mathrm{c}}$ Department of Urology, Onze Lieve Vrouw Hospital, Aalst, Belgium; ${ }^{\mathrm{d}}$ Clinica Urologica I, Azienda Ospedaliera Universitaria Careggi, Università degli studi di Firenze, Firenze, Italy; ${ }^{\mathrm{e}}$ Department of Urology, Università degli studi e Spedali Civili di Brescia, Brescia, Italy; ${ }^{\mathrm{f}}$ Department of Urology, University Hospital Gasthuisberg, Katholieke Universiteit Leuven, Leuven, Belgium

\section{Article info}

Article history:

Accepted February 28, 2019

Associate Editor:

Gianluca Giannarini

\section{Keywords:}

Kidney cancer

Renal cancer

Cardiovascular events

Hypertension

Partial nephrectomy

Radical nephrectomy

\begin{abstract}
Background: Despite better renal function following nephron-sparing surgery (NSS) relative to radical nephrectomy ( $\mathrm{RN}$ ), there is no consensus with respect to the long-term sequelae associated with surgery.

Objective: To investigate the effect of surgery and the temporal pattern of two different cardiovascular event (CVe) categories after NSS versus RN.

Design, setting, and participants: We collected data of 898 patients with cT1-2 N0 M0 renal mass and no history of CVe treated with NSS versus RN. CVe categories were dichotomised in (1) de novo hypertension (HT) and (2) other major cardiovascular events (MCEs).

Outcome measurements and statistical analysis: Multivariable competing regression analyses (MVAs) tested the adjusted effect of surgery type on each CVe category.

Results and limitations: Among patients treated with RN, 38\% of HT events occurred immediately after surgery. Conversely, in NSS counterparts, the onset of HT was diluted over the years after surgery (10\% of HT events in the first $6 \mathrm{mo}$ ). When an MCE was considered, an increasing long-term time-dependent prevalence of the outcome was observed in both groups, with no statistically significantly difference between NSS and RN. At MVA, RN was associated with a higher HT risk (hazard ratio [HR] 2.89; $p=0.006$ ) than but a similar MCE risk (HR $0.85 ; p=0.6$ ) to NSS.

Conclusions: Relative to RN, NSS showed an independent protective effect on HT but not on MCEs. In patients with no history of preoperative HT or MCEs, the onset of HT after $\mathrm{RN}$ is a very early event, due probably to the acute loss of renal parenchyma. This is not the case for the other cardiovascular morbidity, which develops in the long-term period, regardless of the type of surgery performed.

Patient summary: In renal cancer patients without a medical history of cardiopathy, preserving healthy kidney tissue at surgery is associated with a decreased risk of developing postoperative hypertension.

(c) 2019 European Association of Urology. Published by Elsevier B.V. All rights reserved.
\end{abstract}

https://doi.org/10.1016/j.euo.2019.02.006

2588-9311/@ 2019 European Association of Urology. Published by Elsevier B.V. All rights reserved.

\footnotetext{
* Corresponding author. Department of Urology, San Raffaele Scientific Institute, via Olgettina 60, 20132 Milan, Italy. Tel. +39 022643 7286; Fax: +39 0226437298.

E-mail address: capitanio.umberto@hsr.it (U. Capitanio).
} 


\section{Introduction}

The equipoise in oncological outcomes between nephronsparing surgery (NSS) and radical nephrectomy (RN) for patients with clinically localised kidney cancer [1] is well established. For this reason-according to all available international guidelines-if a healthy part of the kidney can be safely spared, the treatment of choice is NSS. Notably, better renal function following NSS represents the most widely accepted clinical benefit of NSS compared with RN [2]. Moreover, it has been suggested that NSS may decrease the risk of end-stage renal disease and cardiovascular events (CVe) after surgery [1-5].

To better understand the natural history of cardiovascular morbidity after surgery, we created a multi-institutional collaboration to investigate the effect of NSS on two specific cardiovascular categories and to analyse the temporal pattern of presentation of all the events.

\section{Patients and methods}

\subsection{Population}

A multi-institutional collaboration of three tertiary care centres enabled the collection into a prospectively maintained database of 2092 consecutive patients diagnosed with a single clinical T1-2 N0 M0 renal mass who underwent elective surgical treatment between 1987 and 2016. To rule out any potential confounding effect of a pre-existing condition of cardiovascular morbidity, we have excluded from our analysis all cases with a previous history of either hypertension (HT) or CVe $(n=1173)$ Patients with missing follow-up information were also excluded $(n=21)$. After these exclusions, the final cohort consisted of 898 patients with cT1-2 N0 M0 renal mass and no baseline CVe.

\subsection{Clinical and pathological evaluation}

Clinical tumour size was calculated based on preoperative imaging and was defined as the greatest tumour diameter in centimetres. The estimated glomerular filtration rate (eGFR) was calculated with the Chronic Kidney Disease Epidemiology Collaboration formula in patients younger than $70 \mathrm{yr}$ old, while the Berlin Initiative Study (BIS1) formula was used for older patients ( $\geq 70 \mathrm{yr}$ ).

\subsection{Outcomes}

The outcomes considered for this study were de novo HT and major cardiovascular events (MCEs). HT was defined as a diagnosis of HT requiring at least one drug, while coronary heart disease, cerebrovascular event, thromboembolic event, dysrhythmias, and peripheral arteriopathy were considered as MCEs. The treating physician or cardiologist defined the need for therapy in case of HT and characterisation of MCE type. Those endpoints were collected by scrutinizing subsequent hospital admission charts or during periodic follow-up visits or, alternatively, by phone call if a patient was referred to another hospital after surgery. In patients with multiple MCE, only the first occurred after surgery was taken into consideration for the purpose of our analysis, to account for the competing nature of the two conditions.

\subsection{Covariates}

Analyses were adjusted for the following covariates: age at surgery, gender, preoperative eGFR, comorbidities (defined according to the
Charlson comorbidity index [CCI]) [6], diabetes, smoking history (active smoker vs former smoker vs no smoking history), clinical tumour size, and year of surgery.

\subsection{Statistical analyses}

Statistical analyses as well as reporting and interpretation of the results were conducted according to established guidelines [7] and consisted of three steps. First, medians and interquartile ranges were reported for continuous variables, or frequencies and proportions were reported for categorical variables. Mann-Whitney and chi-square tests were used to compare the statistical significance of differences in the distribution of continuous and categorical variables, respectively, between cases treated with NSS versus RN.

Second, given the competing nature of the outcomes examined, smoothed Poisson cumulative curves were used to assess the rates of HT and MCEs.

Third, the impact of treatment modality, namely, NSS versus RN, on the study outcomes was estimated using a multivariable competing regression analysis (MVA) after adjustment for all the study covariates. All statistical tests were performed using the RStudio graphical interface v.0.98 for R software environment v.3.0.2 with the following libraries, packages, and scripts: Hmisc, plyr, stats, rms, and cmprsk. All tests were two sided, with a significance level set at $p<0.05$.

\section{Results}

RN and NSS were performed, respectively, in 36\% ( $n=326)$ and $64 \%(n=572)$ of the patients (Table 1$)$. Patients treated with NSS were diagnosed with a smaller tumour (median clinical size 3 vs $6 \mathrm{~cm} ; p<0.0001$ ) and had higher eGFR at baseline (median eGFR 93 vs 87; $p<0.0005$ ) relative to their RN counterparts.

Median follow-up was 58 mo (interquartile range 22115). Figs. 1 and 2 depict the smoothed Poisson cumulative prevalence of HT and MCEs in NSS and RN patients, respectively. The 5-yr HT and MCE rates were, respectively, $3.3 \%$ and $6.3 \%$ in NSS cases versus $5.1 \%$ and $4.2 \%$ in RN cases. In NSS, the onset of HT was diluted over the years after surgery (10\% of all HT events in the first $6 \mathrm{mo}$; Fig. 1, red curve), while $38 \%$ of all HT events in RN occurred in the first 6 mo after surgery (Fig. 2, red curve). Conversely, the vast majority of MCEs accumulate regularly over time, regardless of the type of surgery (Figs. 1 and 2, grey curves). At MVA, after accounting for all the potential confounders, RN was associated with a higher risk of HT (hazard ratio 2.89; 95\% confidence interval $1.35-6.17 ; p=0.006$ ) but a similar MCE risk (hazard ratio 0.85 ; $95 \%$ confidence interval $0.4-1.17$; $p=0.66$ ) relative to NSS (Table 2 ).

\section{Discussion}

NSS is currently the standard of care for patients who are candidates for surgery for a clinical T1 renal mass, when technically feasible $[1,8,9]$. NSS is equivalent to RN in terms of cancer control [10-14] but is associated with better renal function [15,16]. Recent data have suggested a potential benefit in terms of decreasing the risk of subsequent CVe in patients treated with NSS in comparison with RN [1619]. Many mechanisms have been suggested to explain why 


\begin{tabular}{|c|c|c|c|}
\hline Variable & $\begin{array}{c}\text { NSS } \\
(n=572,64 \%)\end{array}$ & $\begin{array}{c}\mathrm{RN} \\
(n=326,36 \%)\end{array}$ & $p$ value \\
\hline Age & & & 0.6 \\
\hline Median & 57 & 56 & \\
\hline IQR & $46-66$ & $47-65$ & \\
\hline Gender & & & 0.2 \\
\hline Male & $361(63)$ & $221(68)$ & \\
\hline Female & $211(37)$ & $105(32)$ & \\
\hline $\mathrm{CCI}$ & & & 0.6 \\
\hline Median & 0 & 0 & \\
\hline $\mathrm{IQR}$ & $0-2$ & $0-1$ & \\
\hline Preoperative eGFR & & & $<0.0005$ \\
\hline Median & 93 & 87 & \\
\hline $\mathrm{IQR}$ & $77-103$ & $71-99$ & \\
\hline Diabetes & & & 0.31 \\
\hline No & $531(93)$ & 309 (95) & \\
\hline Yes & $41(7)$ & $17(5)$ & \\
\hline Smoking status & & & 0.78 \\
\hline No smoking history & $344(60)$ & $198(61)$ & \\
\hline Active smoker & $100(17)$ & $61(19)$ & \\
\hline Former smoker & $128(22)$ & $67(21)$ & \\
\hline Clinical size & & & $<0.0001$ \\
\hline Median & 3 & 6 & \\
\hline $\mathrm{IQR}$ & $2.4-4$ & $4.6-7.5$ & \\
\hline Year of surgery & & & $<0.0001$ \\
\hline Median & 2009 & 2005 & \\
\hline IQR & $2007-2011$ & 1999-2010 & \\
\hline
\end{tabular}

$\mathrm{CCI}=$ Charlson comorbidity index; eGFR = estimated glomerular filtration rate; $\mathrm{IQR}=$ interquartile range; $\mathrm{NSS}=$ nephron-sparing surgery; $\mathrm{RCC}=$ renal cell carcinoma; $\mathrm{RN}=$ radical nephrectomy.

Data are further stratified according to the treatment delivery.

renal function deterioration may be associated with increased cardiovascular morbidity (eg, endothelial dysfunction, increased arterial calcification and stiffness $[20,21]$, increased levels of inflammatory factors [22], high apolipoprotein levels, anaemia [23], and left ventricular hypertrophy [24,25]). Nonetheless, all available data are invariably based on nephropathic patients, living donors, and transplant recipients, and there is a paucity of data in the context of kidney cancer patients. Remarkably, the latter should be regarded as a significant different clinical scenario, due to the differences observed in age and comorbidity profile (eg, diabetes, chronic kidney disease, etc.) that prevent any clinically valid generalisation of the same research findings.

Many previous reports revealed a greater number of cardiovascular events in patients treated with $\mathrm{RN}$ than in those treated with NSS [26-28], although controversies are still there. Huang et al. [27] analysed the Surveillance, Epidemiology, and End Results (SEER)-Medicare cancer registry, and collected data for 2991 patients treated with NSS (19\%) or RN (81\%) between 1995 and 2002. After a mean follow-up period of 43 mo, they reported a $20 \%$ CVe rate after surgery, with a 1.4-fold greater number of events after $\mathrm{RN}(p<0.05)$. However, the results could not be adjusted for potential confounders in patient selection. In this setting, a recent European collaboration conducted a multi-institutional retrospective study, which included 1331 patients with a clinical T1a-T1b N0 M0 renal mass and normal renal function before surgery, confirming a higher CVe risk in RN patients $(n=462,35 \%)$ than in their NSS counterparts ( $n=869,65 \%$ ) [26]. Many criticisms were raised after the publication of the report. Specifically, it was suggested that potential selection biases might have flawed the results due to fact that the curves depicting the rate of CVe between NSS and RN showed an early separation immediately after the surgery. Recently, Yap et al. [29] conducted a large retrospective study in 11937 patients (who underwent either NSS or RN between 1995 and 2010) selected using linked administrative databases in the province of Ontario, Canada. After a median follow-up of 57 mo, they established a benefit of NSS after controlling for competing risks of death for myocardial infarction $(p<0.05)$. However, neither HT nor any other type of CVe, other than myocardial infarction, was considered as an outcome.

To address the need of clarification in this topic, Wang et al. [30] conducted a meta-analysis of renal function and cardiovascular outcomes in patients submitted to either NSS or RN for renal tumour. Overall, 26 studies were pooled for new-onset chronic kidney disease, and six studies were pooled for cardiovascular outcomes; NSS was shown to correlate with a $73 \%$ risk reduction of new-onset chronic kidney disease in all included patients (hazard ratio [HR] $0.27 ; p<0.0001$ ) and a $65 \%$ risk reduction in patients with tumours $>4 \mathrm{~cm}$ (HR 0.35; $p<0.0001)$ compared with RN. It was also shown that there were no significant differences between groups regarding postoperative CVe (HR 0.86; $p=0.2$ ) and cardiovascular death (HR 0.79; $p=0.2$ ).

After accounting for both clinical and tumour characteristics, we were able to demonstrate that NSS independently decreases HT risk relative to RN (RN HR 2.89; 95\% confidence interval $[\mathrm{CI}] 1.35-6.17 ; p=0.006$ ), while no difference was found in MCE risk between the two groups (HR $0.85 ; 95 \%$ CI $0.4-1.17 ; p=0.6$ ). In addition, we demonstrated that while many HT events in RN occur in the first 6 mo after surgery (38\% of all HT events; Fig. 2), in NSS the onset of HT has been diluted over the years after surgery (Fig. 1, red curve). This was not the case for MCEs, where the events occurred later during the follow-up, with an increasing time-dependent prevalence in both the groups (Fig. 1 and 2, grey curves). These findings were further confirmed at MVA, after accounting for potential confounders, where patients who underwent RN were shown to have a higher HT risk (HR 2.89; 95\% CI 1.35-6.17; $p=0.006$ ) than but a similar MCE risk (HR 0.85; 95\% CI 0.41.17; $p=0.66$ ) to their NSS counterparts (Table 2). Since some events were recorded immediately after surgery, we could not include in the adjusted model the variable depicting postoperative eGFR to limit the collinearity and to avoid the inclusion of a variable that might have affected the outcomes only in those patients with no events in the first months after surgery.

One of the possible pathophysiological mechanisms beyond such a finding may be related to the acute loss of half of nephrons, which happens in RN patients. In order to compensate for the parenchymal loss and maintain the preoperative filtration function, the remaining kidney 


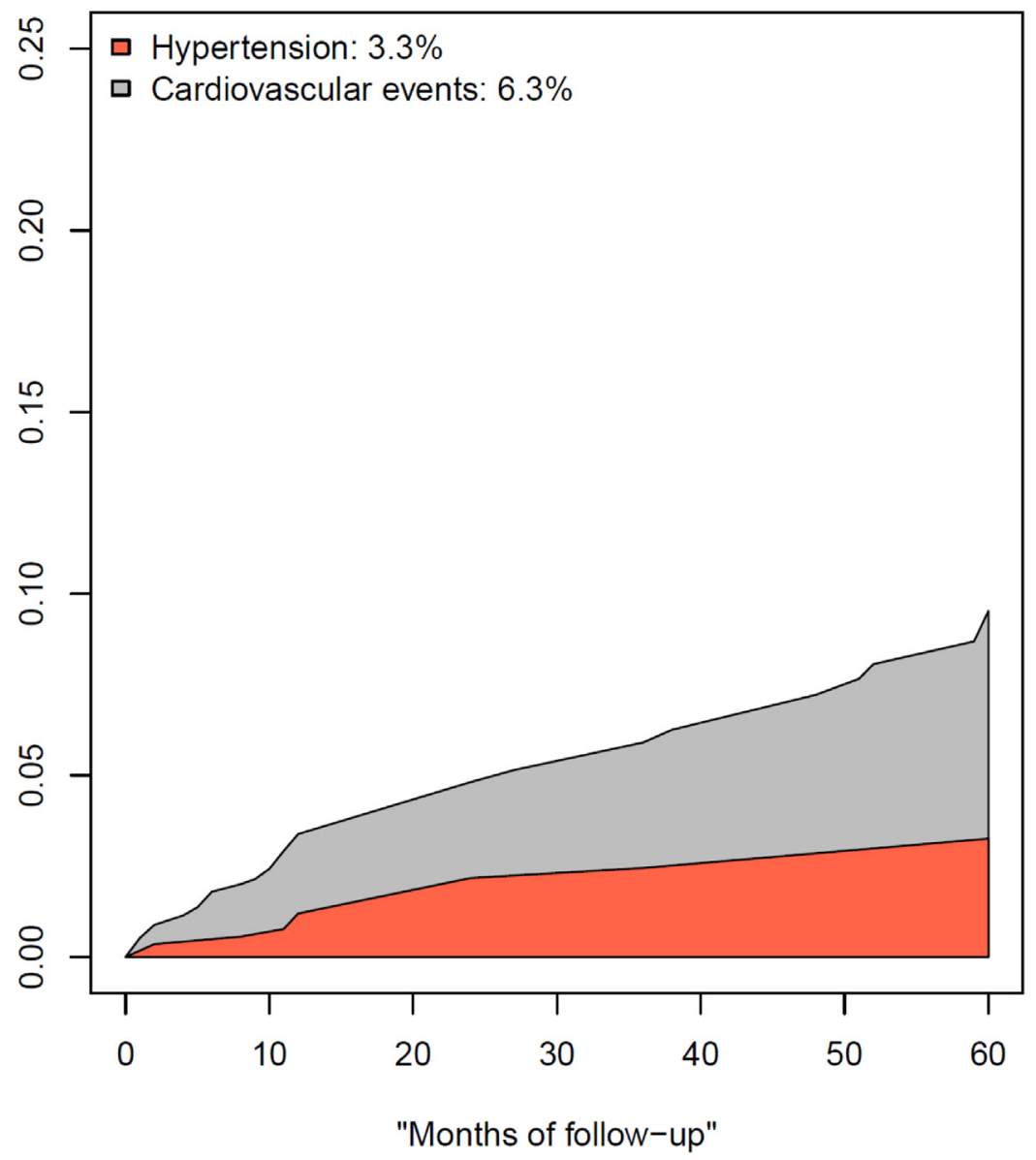

Fig. 1 - Smoothed Poisson cumulative curves depicting hypertension (HT) and major cardiovascular event (MCE) rates in 572 patients diagnosed with a single clinical T1-2 renal mass who underwent NSS between 1987 and 2016 . The 5 -yr HT and MCE rates are $3.3 \%$ and $6.3 \%$, respectively. NSS = nephronsparing surgery.

increases the arterial blood inflow, which induces a compensatory function of the remaining glomeruli [31]. We suppose that this might be achieved through a rapid increase in the renin-angiotensin-aldosterone system (RAAS) activation, with an increased renin production in the kidney. Clearly increased RAAS activation could be the direct culprit of the increased HT risk in these patients, causing the hypertensive state in those already more susceptible to a cardiovascular homeostasis alteration [32]. This hypothesis is further supported by the fact that HT is an early event after surgery: the RAAS activation would be an immediate event after the nephrectomy, and it stands to reason that the HT onset would follow shortly thereafter. Although further research is needed to confirm such a hypothesis, the use of an angiotensin-converting enzyme inhibitor in patients who undergo nephrectomy might represent a potentially useful tool for clinical practice.

Moreover, it was long believed that living kidney donors (LKDs), who are subjected to the same parenchymal loss as patients submitted to RN for kidney cancer, were not exposed to an increased CVe rate $[33,34]$, but there is mounting evidence that these patients are subjected to an increased CVe risk too. Moody et al. [35] demonstrated that unilateral nephrectomy in healthy individuals is associated with structural and functional cardiovascular abnormalities (myocardial deformation, increased aortic stiffness, and increase in left ventricle mass) within $1 \mathrm{yr}$. In addition, Mjøen et al. [36] compared LKDs with healthy controls and observed a significant increase in cardiovascular mortality (HR 1.40; 95\% CI $1.03-1.91 ; p=0.03)$. We suppose that in LKDs, cardiovascular alteration, and following increased CVe risk, might recognise the same causes as those in individuals submitted to RN, due to the fact that both populations undergo a sudden nephron loss [37]. In case of surgery for kidney tumour, such mechanisms are further augmented by the fact that kidney cancer patients are older and more comorbid than their LKD counterparts, and these aspects are well-known detrimental factors for the development of HT and MCEs.

For the first time, our study provides an adjusted analysis of the timing and risk of development of HT and MCEs after NSS or RN. We demonstrated a different temporal pattern of presentation between NSS and RN for HT development, which suggests novel pathophysiological mechanisms beyond the functional outcomes of kidney tumour patients treated with surgery. Besides these merits, the major 


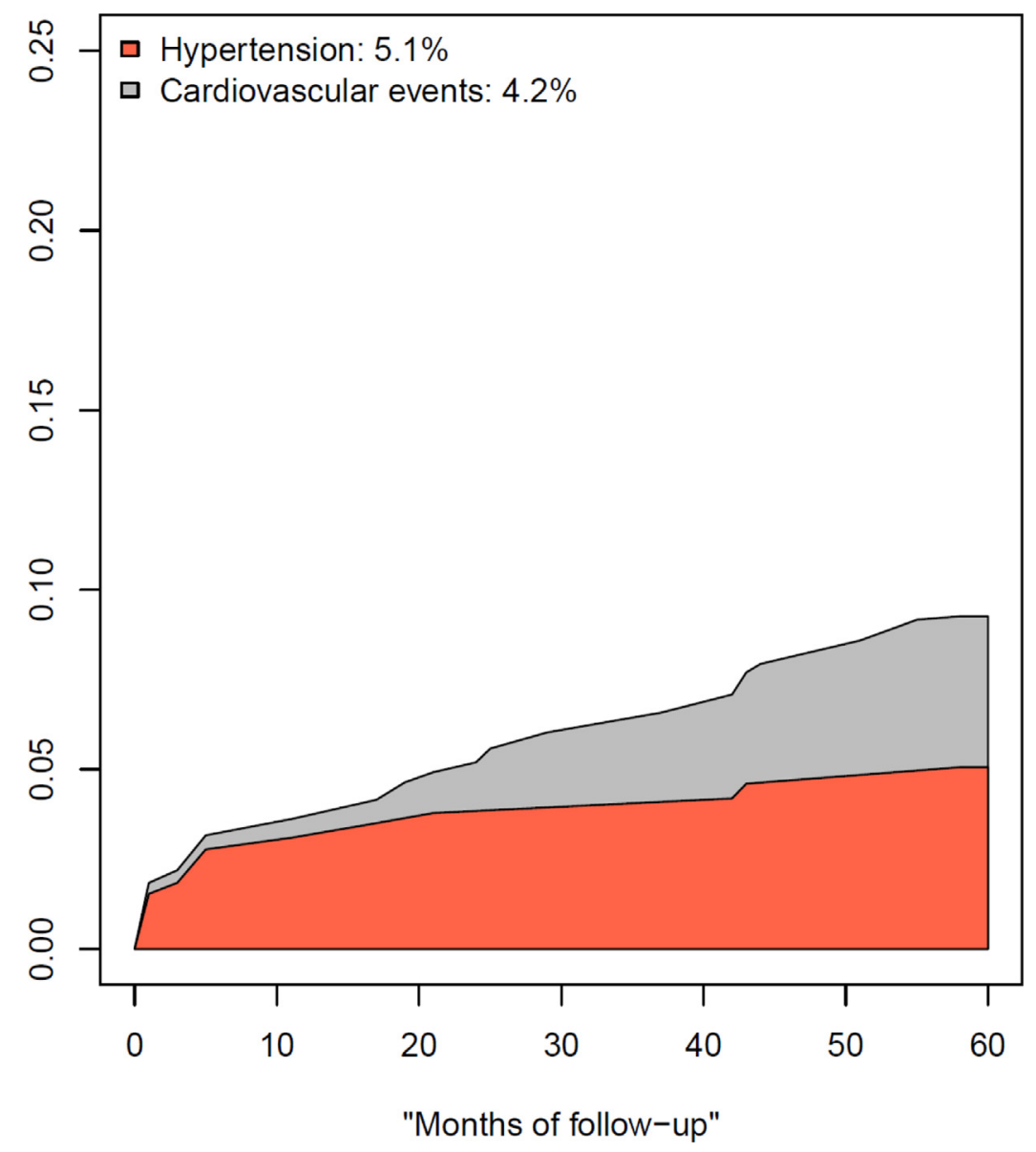

Fig. 2 - Smoothed Poisson cumulative curves depicting hypertension (HT) and major cardiovascular event (MCE) rates in 326 patients diagnosed with a single clinical T1-2 renal mass who underwent $\mathrm{RN}$ between 1987 and 2016 . The 5 -yr $\mathrm{HT}$ and $\mathrm{MCE}$ rates are $5.1 \%$ and $4.2 \%$, respectively. RN = radical nephrectomy.

Table 2 - Multivariable competing risk regression analysis addressing the effect of NSS versus RN on the risk of developing HT or MCEs in patients with no cardiovascular history after adjustment for clinical and pathological confounders

\begin{tabular}{|c|c|c|c|c|}
\hline \multirow[t]{2}{*}{ Predictors } & \multicolumn{2}{|c|}{ MVA predicting HT } & \multicolumn{2}{|c|}{ MVA predicting MCE } \\
\hline & HR (95\% CI) & $p$ value & $\mathrm{HR}(95 \% \mathrm{CI})$ & $p$ value \\
\hline \multicolumn{5}{|l|}{ Approach } \\
\hline NSS & Ref. & & Ref. & \\
\hline RN & 2.89 (1.35-6.17) & 0.006 & $0.85(0.4-1.78)$ & 0.7 \\
\hline Age (yr) & $1.02(0.99-1.05)$ & 0.2 & $1.04(0.99-1.08)$ & 0.09 \\
\hline \multicolumn{5}{|l|}{ Gender } \\
\hline Male & Ref. & & Ref. & \\
\hline Female & $1.16(0.63-2.16)$ & 0.6 & $0.71(0.35-1.42)$ & 0.3 \\
\hline $\mathrm{CCI}$ & $1.02(0.73-1.44)$ & 0.9 & $1.07(0.78-1.48)$ & 0.7 \\
\hline Preoperative eGFR & $0.98(0.96-1)$ & 0.1 & $0.99(0.97-1)$ & 0.09 \\
\hline \multicolumn{5}{|l|}{ Diabetes } \\
\hline No & Ref. & & Ref. & \\
\hline Yes & $0.84(0.23-3.14)$ & 0.8 & $2.16(0.86-5.43)$ & 0.1 \\
\hline \multicolumn{5}{|l|}{ Smoking status } \\
\hline No smoking history & Ref. & & Ref. & \\
\hline Active smoker & $0.7(0.3-1.64)$ & 0.4 & $1(0.47-2.12)$ & 0.9 \\
\hline Former smoker & $0.72(0.31-1.67)$ & 0.4 & $0.92(0.45-1.9)$ & 0.8 \\
\hline Clinical size & $0.94(0.82-1.07)$ & 0.3 & $0.98(0.84-1.15)$ & 0.8 \\
\hline Year of surgery & $1.16(1.08-1.25)$ & 0.0006 & $1.09(1.02-1.18)$ & 0.01 \\
\hline
\end{tabular}

$\mathrm{CCI}=$ Charlson comorbidity index; $\mathrm{CI}=$ confidence interval; eGFR = estimated glomerular filtration rate; $\mathrm{HR}$ = hazard ratio; $\mathrm{HT}$ = hypertension; $\mathrm{MCE}=$ major cardiovascular event; MVA = multivariable analyses; NSS = nephron-sparing surgery; Ref. = reference; $\mathrm{RN}$ = radical nephrectomy. limitation is related to the nonrandomised nature of the comparison. Since it is possible that the characteristics of the patients (CCI, presence of HT or diabetes, etc.) treated with NSS and RN have been changing over the years, and that the accuracy of the treating physician to detect de novo HT or other MCEs has been improving over the last decade, we decided to adjust all the analyses for the year of surgery to correct them for these possible aspects. Moreover, within this data source, we could not test the mutual causal relationship between the postoperative renal function detriment and the risk of CVe. Finally, as was the case for most data available on the topic, the results could not be adjusted for other important risk factors for cardiovascular disease such as hyperlipidaemia, exercise activity, and alcohol use.

\section{Conclusions}

In renal cell carcinoma patients without preoperative cardiovascular morbidity, the effect of surgical approach varies according to the type of CVe that is considered. Relative to RN, NSS showed an independent protective effect on HT but not on MCEs. In patients with no history of preoperative HT or MCEs, the onset of HT after RN is an early event, due probably to the acute loss of renal parenchyma. 
Author contributions: Umberto Capitanio had full access to all the data in the study and takes responsibility for the integrity of the data and the accuracy of the data analysis.

Study concept and design: Capitanio, Larcher.

Acquisition of data: Capitanio, Larcher, Cianflone, Trevisani, Nini, Mottrie, Mari, Campi, Tellini, Briganti, Veccia, Van Poppel, Carini, Simeone, Salonia, Minervini, Antonelli, Montorsi, Bertini.

Analysis and interpretation of data: Capitanio, Larcher, Cianflone, Trevisani.

Drafting of the manuscript: Capitanio, Larcher, Cianflone, Trevisani.

Critical revision of the manuscript for important intellectual content: Capitanio, Larcher, Cianflone, Trevisani, Nini, Mottrie, Mari, Campi, Tellini, Veccia, Van Poppel, Carini, Simeone, Salonia, Minervini, Antonelli, Montorsi, Bertini.

Statistical analysis: Capitanio, Larcher, Cianflone.

Obtaining funding: None.

Administrative, technical, or material support: None.

Supervision: Capitanio, Larcher, Cianflone, Trevisani, Nini, Mottrie, Mari, Campi, Tellini, Veccia, Van Poppel, Carini, Simeone, Salonia, Minervini, Antonelli, Montorsi, Bertini.

Other: None.

Financial disclosures: Umberto Capitanio certifies that all conflicts of interest, including specific financial interests and relationships and affiliations relevant to the subject matter or materials discussed in the manuscript (eg, employment/affiliation, grants or funding, consultancies, honoraria, stock ownership or options, expert testimony, royalties, or patents filed, received, or pending), are the following: None.

Funding/Support and role of the sponsor: Research activity in the field of kidney cancer at Urological Research Institute, IRCCS Ospedale San Raffaele, is supported by an unrestricted grant from Recordati.

\section{References}

[1] Ljungberg B, Bensalah K, Bex A, et al. EAU guidelines on renal cell carcinoma. Arnhem, The Netherlands: EAU Guidelines Office; 2018. http://uroweb.org/guidelines/compilations-of-all-guidelines/

[2] Kim SP, Campbell SC, Gill I, et al. Collaborative review of risk benefit trade-offs between partial and radical nephrectomy in the management of anatomically complex renal masses. Eur Urol 2017;72:64-75.

[3] Tourojman M, Kirmiz S, Boelkins B, et al. Impact of reduced glomerular filtration rate and proteinuria on overall survival of patients with renal cancer. J Urol 2016;195:588-93.

[4] O'Donnell K, Tourojman M, Tobert CM, et al. Proteinuria is a predictor of renal functional decline in patients with kidney cancer. J Urol 2016;196:658-63.

[5] Capitanio U, Montorsi F. Renal cancer. Lancet 2016;387:894-906.

[6] Charlson ME, Pompei P, Ales KL, MacKenzie CR. A new method of classifying prognostic comorbidity in longitudinal studies: development and validation. J Chronic Dis 1987;40:373-83.

[7] Vickers AJ, Sjoberg DD. Guidelines for reporting of statistics in European Urology. Eur Urol 2015;67:181-7.

[8] Escudier B, Porta C, Schmidinger M, et al. Renal cell carcinoma: ESMO clinical practice guidelines for diagnosis, treatment and follow-up. Ann Oncol 2016;27(Suppl. 5):v58-68.

[9] Campbell S, Uzzo RG, Allaf ME, et al. Renal mass and localized renal cancer: AUA guideline. J Urol 2017;198:520-9.

[10] Van Poppel H, Da Pozzo L, Albrecht W, et al. A prospective, randomised EORTC intergroup phase 3 study comparing the oncologic outcome of elective nephron-sparing surgery and radical nephrectomy for low-stage renal cell carcinoma. Eur Urol 2011;59:543-52.
[11] Antonelli A, Ficarra V, Bertini R, et al. Elective partial nephrectomy is equivalent to radical nephrectomy in patients with clinical T1 renal cell carcinoma: results of a retrospective, comparative, multi-institutional study. BJU Int 2012;109:1013-8.

[12] Capitanio U, Zini L, Perrotte P, et al. Cytoreductive partial nephrectomy does not undermine cancer control in metastatic renal cell carcinoma: a population-based study. Urology 2008;72:1090-5.

[13] Crépel M, Jeldres C, Sun M, et al. A population-based comparison of cancer-control rates between radical and partial nephrectomy for T1A renal cell carcinoma. Urology 2010;76:883-8.

[14] Zini L, Perrotte P, Capitanio U, et al. Radical versus partial nephrectomy: effect on overall and noncancer mortality. Cancer 2009;115:1465-71.

[15] Scosyrev E, Messing EM, Sylvester R, Campbell S, Van Poppel H. Renal function after nephron-sparing surgery versus radical nephrectomy: results from EORTC randomized trial 30904. Eur Urol 2014;65:372-7.

[16] Scosyrev E, Messing E, Campbell S. Radical versus partial nephrectomy for a small renal mass: does saving nephrons save lives? Expert Rev Anticancer Ther 2013;13:1349-51.

[17] Ivanovski O, Nikolov I, Davceva O, Gjorgjievska K, Janevska V, Petrusevska G. Partial nephrectomy may protect from future cardiovascular disease-first experimental evidence. Eur Urol Suppl 2013;12:e1261, S153.

[18] Go AS, Chertow GM, Fan D, McCulloch CE, Hsu CY. Chronic kidney disease and the risks of death, cardiovascular events, and hospitalization. N Engl J Med 2004;351:1296-305.

[19] Takeshita H, Yokoyama M, Fujii Y, et al. Impact of renal function on cardiovascular events in patients undergoing radical nephrectomy for renal cancer. Int J Urol 2012;19:722-8.

[20] Raggi P, Boulay A, Chasan-Taber S, et al. Cardiac calcification in adult hemodialysis patients. A link between end-stage renal disease and cardiovascular disease? J Am Coll Cardiol 2002;39:695-701.

[21] London GM, Guérin AP, Marchais SJ, Métivier F, Pannier B, Adda H. Arterial media calcification in end-stage renal disease: impact on all-cause and cardiovascular mortality. Nephrol Dial Transplant 2003;18:1731-40.

[22] Shlipak MG, Fried LF, Crump C, et al. Elevations of inflammatory and procoagulant biomarkers in elderly persons with renal insufficiency. Circulation 2003;107:87-92.

[23] Hsu C-Y, McCulloch CE, Curhan GC. Iron status and hemoglobin level in chronic renal insufficiency. J Am Soc Nephrol 2002;13:2783-6.

[24] Levin A, Djurdjev O, Barrett B, et al. Cardiovascular disease in patients with chronic kidney disease: getting to the heart of the matter. Am J Kidney Dis 2001;38:1398-407.

[25] Blacher J, Safar ME, Guerin AP, Pannier B, Marchais SJ, London GM. Aortic pulse wave velocity index and mortality in end-stage renal disease. Kidney Int 2003;63:1852-60.

[26] Capitanio U, Terrone C, Antonelli A, et al. Nephron-sparing techniques independently decrease the risk of cardiovascular events relative to radical nephrectomy in patients with a T1a-T1b renal mass and normal preoperative renal function. Eur Urol 2015;67:683-9.

[27] Huang WC, Elkin EB, Levey AS, Jang TL, Russo P. Partial nephrectomy versus radical nephrectomy in patients with small renal tumors-is there a difference in mortality and cardiovascular outcomes? J Urol 2009;181:55-61, [discussion 61-2].

[28] Weight CJ, Larson BT, Fergany AF, et al. Nephrectomy induced chronic renal insufficiency is associated with increased risk of cardiovascular death and death from any cause in patients with localized cT1b renal masses. J Urol 2010;183:1317-23.

[29] Yap SA, Finelli A, Urbach DR, Tomlinson GA, Alibhai SMH. Partial nephrectomy for the treatment of renal cell carcinoma (RCC) and the risk of end-stage renal disease (ESRD). BJU Int 2015;115:897-906. 
[30] Wang Z, Wang G, Xia Q, et al. Partial nephrectomy vs. radical nephrectomy for renal tumors: a meta-analysis of renal function and cardiovascular outcomes. Urol Oncol Semin Orig Investig 2016;34:533.e11-e.

[31] Pluznick JL. Renal and cardiovascular sensory receptors and blood pressure regulation. Am J Physiol Renal Physiol 2013;305:F439-44.

[32] Te Riet L, van Esch JHM, Roks AJM, van den Meiracker AH, Danser AHJ. Hypertension: renin-angiotensin-aldosterone system alterations. Circ Res 2015;116:960-75.

[33] Fehrman-Ekholm I, Elinder CG, Stenbeck M, Tydén G, Groth CG. Kidney donors live longer. Transplantation 1997;64:976-8.
[34] Fournier C, Pallet N, Cherqaoui Z, et al. Very long-term follow-up of living kidney donors. Transpl Int 2012;25:385-90.

[35] Moody WE, Ferro CJ, Edwards NC, et al. Cardiovascular effects of unilateral nephrectomy in living kidney donors. Hypertension 2016;67:368-77.

[36] Mjøen G, Hallan S, Hartmann A, et al. Long-term risks for kidney donors. Kidney Int 2014;86:162-7.

[37] Antonelli A, Minervini A, Sandri M, et al. Below safety limits, every unit of glomerular filtration rate counts: assessing the relationship between renal function and cancer-specific mortality in renal cell carcinoma. Eur Urol 2018;74:661-7. 